\title{
Silicon pore optics x-ray mirror development for the Athena telescope
}

Collon, Maximilien J.; Babic, Ljubiša; Barrière, Nicolas M.; Bayerle, Alex; Castiglione, Luigi; Eenkhoorn, Noë; Girou, David; Günther, Ramses; Hauser, Enrico; Jenkins, Yvette

Total number of authors:

48

Published in:

Proceedings of SPIE

Link to article, DOI:

$10.1117 / 12.2593505$

Publication date:

2021

Document Version

Publisher's PDF, also known as Version of record

Link back to DTU Orbit

Citation (APA):

Collon, M. J., Babic, L., Barrière, N. M., Bayerle, A., Castiglione, L., Eenkhoorn, N., Girou, D., Günther, R., Hauser, E., Jenkins, Y., Landgraf, B., Keek, L., Okma, B., Mendoza Serrano, G., Thete, A., Vacanti, G., Verhoeckx, S., Vervest, M., Voruz, L., ... Ball, K. (2021). Silicon pore optics x-ray mirror development for the Athena telescope. In S. L. O'Dell, J. A. Gaskin, \& G. Pareschi (Eds.), Proceedings of SPIE: Optics for EUV, XRay, and Gamma-Ray Astronomy X [1182206] SPIE - International Society for Optical Engineering. Proceedings of SPIE - The International Society for Optical Engineering Vol. 11822 https://doi.org/10.1117/12.2593505

\section{General rights}

Copyright and moral rights for the publications made accessible in the public portal are retained by the authors and/or other copyright owners and it is a condition of accessing publications that users recognise and abide by the legal requirements associated with these rights.

- Users may download and print one copy of any publication from the public portal for the purpose of private study or research.

- You may not further distribute the material or use it for any profit-making activity or commercial gain

- You may freely distribute the URL identifying the publication in the public portal 


\title{
Silicon Pore Optics X-ray mirror development for the ATHENA telescope
}

\author{
Maximilien J. Collon*1, Ljubiša Babić ${ }^{1}$, Nicolas M. Barrière ${ }^{1}$, Alex Bayerle ${ }^{1}$, Luigi Castiglione ${ }^{1}$, \\ Noë Eenkhoorn ${ }^{1}$, David Girou ${ }^{1}$, Ramses Günther ${ }^{1}$, Enrico Hauser ${ }^{1}$, Yvette Jenkins ${ }^{1}$, Boris Landgraf ${ }^{1}$, \\ Laurens Keek ${ }^{1}$, Ben Okma ${ }^{1}$, Gregorio Mendoza Serrano ${ }^{1}$, Aniket Thete ${ }^{1}$, Giuseppe Vacanti ${ }^{1}$, \\ Sjoerd Verhoeckx ${ }^{1}$, Mark Vervest ${ }^{1}$, Luc Voruz ${ }^{1}$, Marco W. Beijersbergen ${ }^{1}$, \\ Marcos Bavdaz ${ }^{2}$, Eric Wille ${ }^{2}$, Ivo Ferreira ${ }^{2}$, Sebastiaan Fransen ${ }^{2}$, Brian Shortt ${ }^{2}$, \\ Mark Olde Riekerink ${ }^{3}$, Jeroen Haneveld ${ }^{3}$, Arenda Koelewijn ${ }^{3}$, Maurice Wijnperle ${ }^{3}$, \\ Jan-Joost Lankwarden ${ }^{3}$, Bart Schurink ${ }^{3}$, Ronald Start ${ }^{3}$, \\ Coen van Baren ${ }^{4}$, Paul Hieltjes ${ }^{4}$, Jan Willem den Herder ${ }^{4,10}$, \\ Evelyn Handick ${ }^{5}$, Michael Krumrey, \\ Miranda Bradshaw ${ }^{6}$, Vadim Burwitz ${ }^{6}$, \\ Sonny Massahi ${ }^{7,11}$, Sara Svendsen ${ }^{7}$, Desirée Della Monica Ferreira ${ }^{7,11}$, Finn E. Christensen ${ }^{7,11}$, \\ Giuseppe Valsecchi ${ }^{8}$, \\ Geeta Kailla ${ }^{9}$, William Mundon ${ }^{9}$, Gavin Phillips ${ }^{9}$, Kevin Ball ${ }^{9}$ \\ ${ }^{1}$ cosine, Oosteinde 36, 2361 HE Warmond, The Netherlands \\ ${ }^{2}$ European Space Agency, ESTEC, Keplerlaan 1, 2200 AG Noordwijk, The Netherlands \\ ${ }^{3}$ Micronit B.V., Colosseum 15, 7521 PV Enschede, The Netherlands \\ ${ }^{4}$ SRON, Sorbonnelaan 2, 3584 CA Utrecht, The Netherlands \\ ${ }^{5}$ Physikalisch-Technische Bundesanstalt (PTB), Abbestr. 2-12, 10587 Berlin, Germany \\ ${ }^{6}$ MPI f. extraterrestrische Physik, Giessenbachstrasse 1, 85748 Garching, Germany \\ ${ }^{7}$ DTU Space, Technical University of Denmark, Building 327, DK - 2800 Kgs. Lyngby, Denmark \\ ${ }^{8}$ Media Lario S.r.l., Località Pascolo, 23842 Bosisio Parini(LC), Italy \\ ${ }^{9}$ Teledyne imaging Ltd., 106 Waterhouse Lane, Chelmsford, Essex CM1 2QU, England \\ ${ }^{10}$ Anton Pannekoek Institute, University of Amsterdam, 1090 GE Amsterdam, the Netherlands \\ ${ }^{11}$ CHEXS, Diplomvej 373B, 2800 Kgs. Lyngby, Denmark
}

\begin{abstract}
ATHENA, the largest space-based X-ray telescope to be flown by the European Space Agency, uses a new modular technology to assemble its $2.5 \mathrm{~m}$ diameter lens. The lens will consist of several hundreds of smaller X-ray lenslets, called mirror modules, which each consist of up to 76 stacked mirror pairs. Those mirror modules are arranged in circles in a large optics structure and will focus X-ray photons with an energy of 0.5 to $10 \mathrm{keV}$ at a distance of $12 \mathrm{~m}$ onto the detectors of ATHENA. The point-spread function (PSF) of the optics shall achieve a half-energy width (HEW) of 5", with an effective area of about $1.4 \mathrm{~m}^{2}$ at an energy of $1 \mathrm{keV}$, corresponding to several hundred $\mathrm{m}^{2}$ of super-polished mirrors with a roughness of about $0.3 \mathrm{~nm}$ and a thickness of down to $110 \mu \mathrm{m}$. Silicon Pore Optics (SPO), using the highest-grade double-side polished $300 \mathrm{~mm}$ wafers commercially available, have been invented to enable such next generation X-ray telescopes. SPO allows the cost-effective production of high-resolution, large area X-ray optics by using all the advantages that mono-crystalline silicon and the mass production processes of the semiconductor industry provide. SPO has also shown to be a versatile technology that can be further developed for gamma-ray optics, medical applications and for materials research. This paper will present the status of the technology and of the mass production capabilities, show latest performance results and discuss the next steps in the development.
\end{abstract}

Keywords: X-ray optics, X-ray astronomy, silicon, wafer, stack, pore optics, X-ray telescopes, ATHENA, ARCUS, SPO

*m.collon@cosine.nl

Optics for EUV, X-Ray, and Gamma-Ray Astronomy X, edited by Stephen L. O'Dell,

Jessica A. Gaskin, Giovanni Pareschi, Proc. of SPIE Vol. 11822, 1182206 · C

2021 SPIE · CCC code: 0277-786X/21/\$21 - doi: 10.1117/12.2593505 


\section{INTRODUCTION}

ATHENA [1], the second L-class mission selected by the European Space Agency (ESA) within its Cosmic Vision 2015 2025 plan [2], will be the largest X-ray telescope built to date. It will study the 'Hot and Energetic Universe' and essentially consists of a $2.5 \mathrm{~m}$ diameter lens and two large detectors, a Wide-Field Imager (WFI) [3] and a cryogenic imaging spectrometer called the X-ray Integral Field Unit (XIFU) [4]. ESA is currently studying the mission and is now in Phase B1 of that process [5]. The next gate is the Mission Adoption Review (MAR), scheduled for November 2022, to put the mission on schedule for launch in 2033.

Apart from the detectors, ATHENA's optics is a new and mission enabling element. To achieve the scientific goals requires [6] a $12 \mathrm{~m}$ focal length lens that can provide an effective area of about $1.4 \mathrm{~m}^{2}$ at an energy of $1 \mathrm{keV}$ and that can achieve an angular resolution of 5" half-energy width (HEW). When first design studies for ATHENA were performed in the early years of 2000 the unprecedented demand for a combination of large area and high-resolution X-ray optics was outside of the realm of then existing technologies [7]. To provide such optics, ESA therefore started in 2005 the development of a light-weight X-ray lens technology, now called Silicon Pore Optics (SPO) [8]. With the selection of ATHENA in 2014 the SPO development efforts intensified, in order to reach a sufficient Technology Readiness Level (TRL) of 5-6, as required to pass the MAR.

SPO allows assembling the ATHENA optics in a modular way, by using 600 mirror modules, essentially small X-ray lenslets [8], that are inserted, aligned and fixed into an optical bench. Each mirror module consists of pairs of stacks of coated mirror plates made from commercial super-polished silicon wafers [9]. The total optics will include close to 90.000 of such mirror surfaces, with a total area of $\sim 300 \mathrm{~m}^{2}$ and a surface roughness of $\sim 0.3 \mathrm{~nm}$ rms. To produce such large amounts of individual mirrors and to assemble hundreds of mirror modules [10] requires a mass production approach that so far has been unheard of in scientific astrophysical missions. Demonstration of the capability to produce the SPO mirror modules efficiently and with high quality is the key criterion for the ESA ATHENA adoption approach. This paper summarises the status of reading this new technology for the ATHENA mission.

\section{PREPARING THE MASS PRODUCTION OF SPO}

The production of the ATHENA optics is split into 4 major steps: (1) production of the mirror plates, (2) stacking of the mirrors, (3) assembly of mirror modules and (4) integration of the mirror modules into the optics structure. cosine will be responsible for all steps up to mirror module level and provide those then to Media Lario S.r.l. in Italy for integration into the optical bench [11].

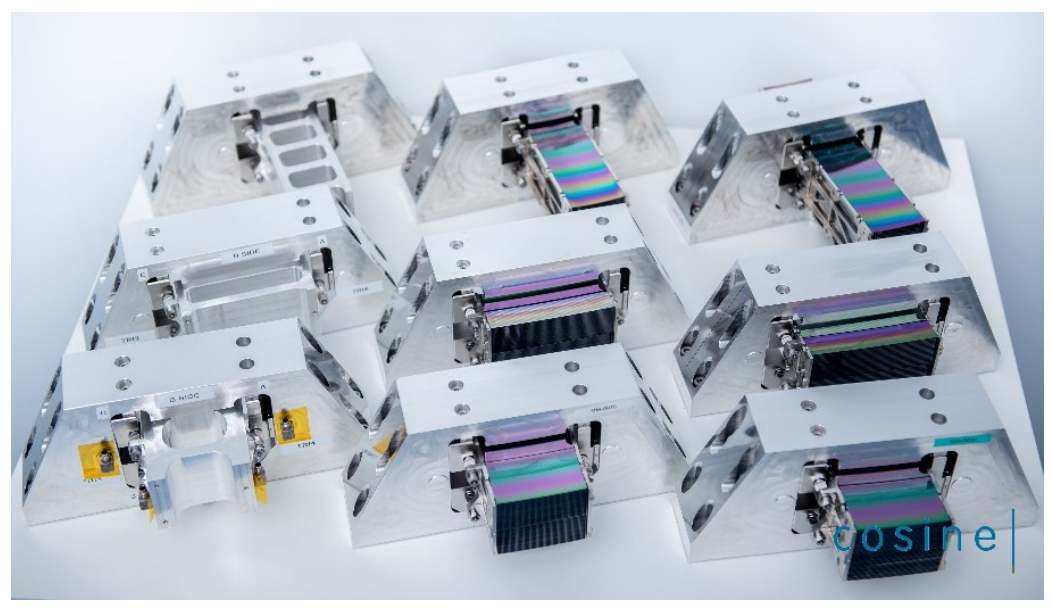

Figure 1: Inner, middle and outer radius (from back to front) SPO mirror modules being prepared for vibration testing. The left most row shows mass dummies used to calibrate the test equipment.

All critical elements of the entire production chain have already been demonstrated individually as part of the ongoing technology development program of ESA, using early development samples in small quantities. In order to avoid the typical problems when scaling up from laboratory scale to mass production during the transition of the ATHENA project 
into phase C (building), a soft ramp-up approach has been chosen. This means that industrial grade equipment is procured already now in phase B, installed, commissioned and used to speed up and gradually increase the production rates. This approach ensures that the adoption review process can also be provided with evidence that a production rate of two mirror modules per day [10], as required to keep the ATHENA schedule [5], is realistic and feasible.

\section{Mirror plates}

Building the flight model of ATHENA starts with sourcing $~ 15.000$ super-polished $300 \mathrm{~mm}$ wafers [12]. Such an amount, while large for scientific customers, is only a minor part of the 600.000 wafers that are produced each month world-wide for the semiconductor industry. The wafers will then be further polished by an ion-beam figuring (IBF) process to mill a wedge into the silicon, as required to later form a focusing optics. This is a change of the so-far used wet chemical wedging process, which shall allow to further tighten the production parameters and result in an improved optics performance. We are currently procuring one of the first fully automated $300 \mathrm{~mm}$ IBF machines that can load wafer cassettes and process those. The machine will be installed late 2021 in the cosine premises in Warmond and we expect to require only one additional machine to process all of the wafers required for ATHENA.

The processed wafers are then sent to the two plate suppliers (Micronit microtechnologies in the Netherlands and Teledyne $\mathrm{e} 2 \mathrm{v}$ in the United Kingdom) for further processing. Both suppliers have installed automated wetbenches for the chemical processes and automated dicing saws for the ribbing and dicing processes required to turn the wafers into mirror plates (see figure 2).
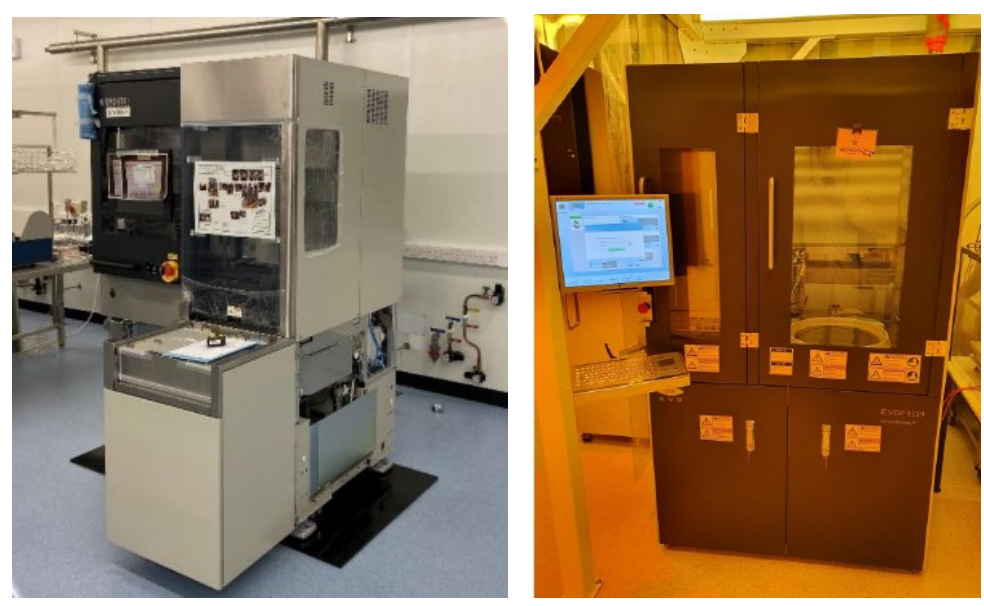

Figure 2: Automated wafer dicing and ribbing machine, as installed at both plate suppliers (left). The right shows the first industrial spray-coater after installation at Micronit.

This equipment already allows producing and delivering several thousands of plates per year and which was used in the past two years to exercise all aspects of the production. The pilot production at small quantities revealed many aspects and difficulties that have been improved upon over the last two years: The plate specification and acceptance criteria have been detailed and iterated to find a balance between yield (cost) and performance. The packaging and shipment containers have been designed, prototyped, tested and improved iteratively, to maximise cleanliness and improve efficiency both at packaging and un-packaging sites. The plate production for more standard plate types has been transferred from the engineering to the production departments, which yields many steps where small improvements result in significant cost savings and/or quality improvements. Micronit has also just installed a first industrial spray-coater (see figure 2) to develop the mass-production resist deposition process for pattering the mirror plates such, that the later metal coating will not be on areas where plate-to-plate bonding will take place. 

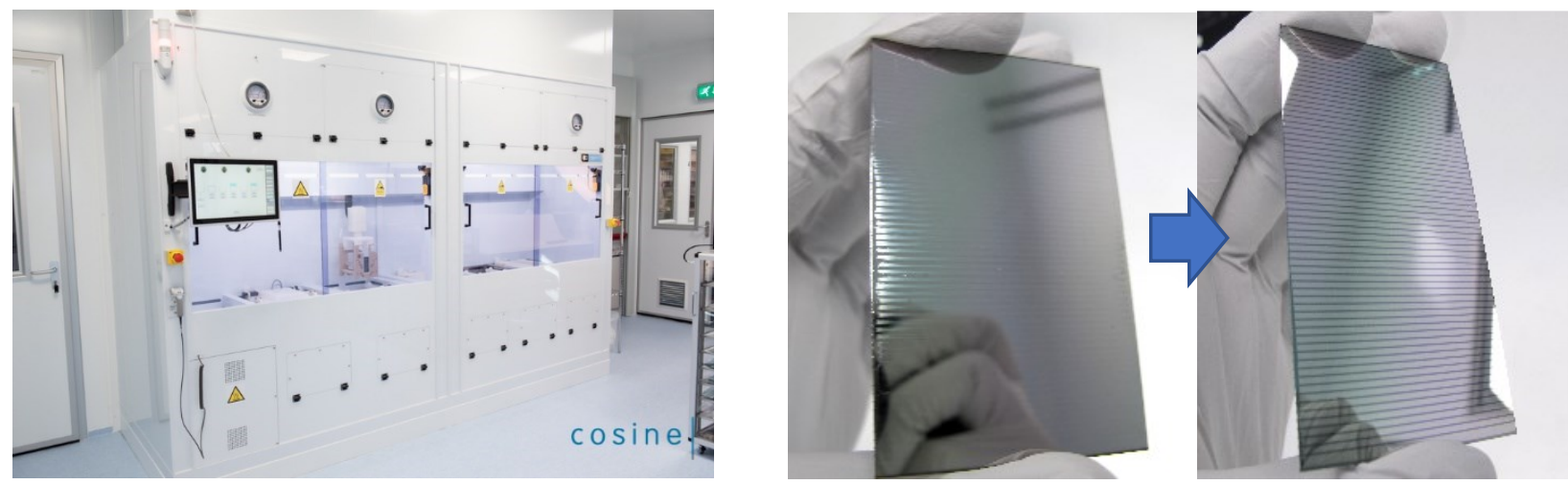

Figure 3: The left photograph shows the fully automated cleaning and lift-off bench, that has recently been installed at cosine. The right two photographs show a comparison of an iridium coated plate before and after lift-off.

Once the plates have been delivered to cosine, they need to be further processed. The first step is an incoming inspection. Plate inspection software applications have been developed, such that technicians can efficiently communicate inspection results and store the data for analysis and statistics in a centralized database. In 2020 additional metrology has been developed to measure the total thickness variation of the plates (one of the performance drivers of the ATHENA optics) and since available, has resulted in improvements to the plate wedge production and to the selection of mirror plates for stacking.

The next step is to clean the plates, using a newly installed fully automated wetbench (see figure 3 ). This wetbench performs cleaning and drying of plates according to stored recipes and includes concentration monitoring of all chemicals, which allows to control the filling and spiking to percent levels. Due to the automation, the timing for heating up baths, inserting containers with plates and running cleaning cycles can be repeated with seconds accuracy. The automation also off-loads the technicians that now only have to load and unload the containers.
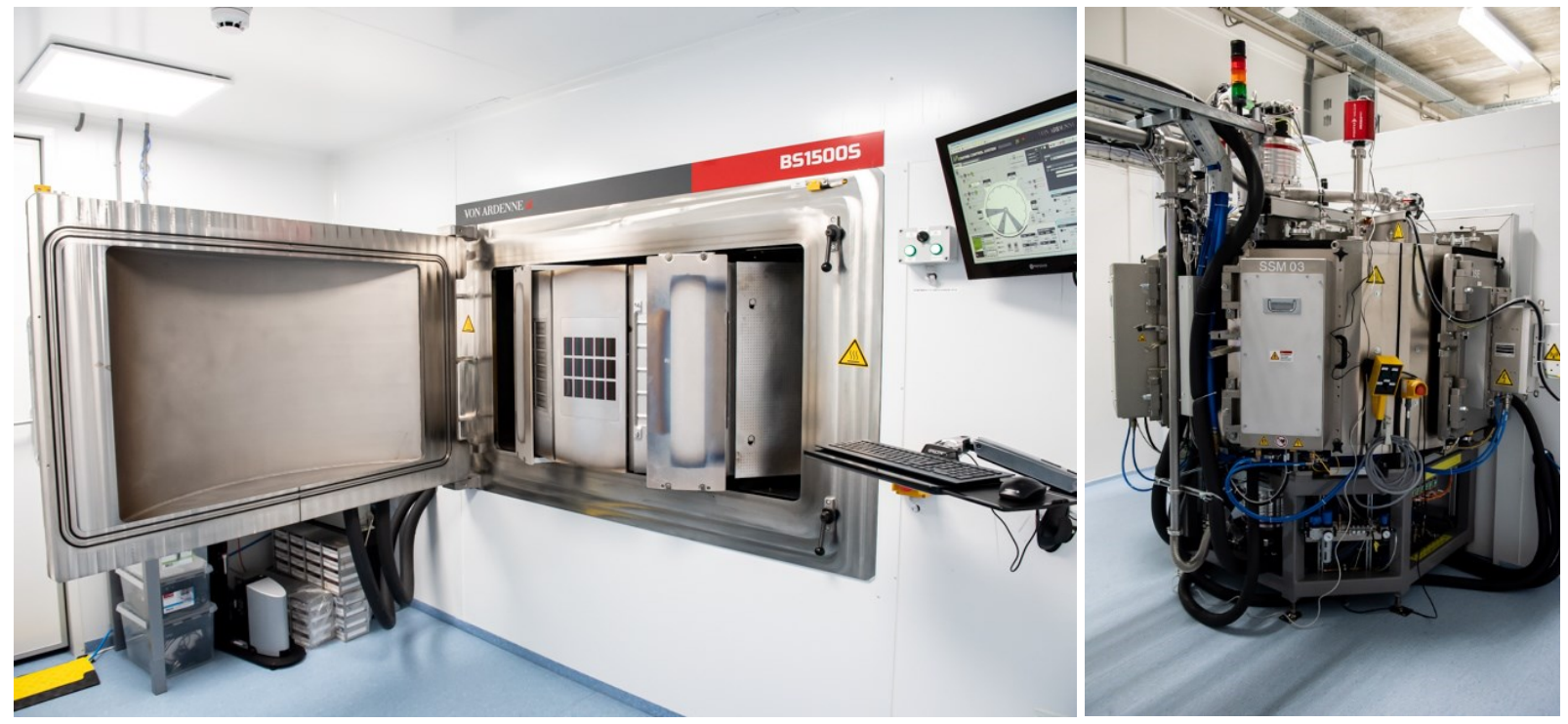

Figure 4: The left photograph shows the industrial magntetron drum coating machine opened from the cleanroom side for loading. A sample holder with 5x3 SPO plates just has been loaded. The system is completely computer controlled and the operator can load coating recipes using a interface mounted next to it. The right photograph shows the other side of the machine, with three magnetrons and a plasma cleaning system.

The cleaned plates are then transferred into carriers to load them into an industrial magnetron sputtering drum coating machine (see figure 4). This machine has been installed in 2019 [13] and has in the last months been upgraded to include three magnetrons that can sputter sequentially materials such as iridium, chromium, silicon carbide, boron carbide or 
carbon. The coating machine is also fully automated and coating recipes are stored and can be run without the intervention of operators. The machine includes an plasma system [14] to clean the mirror before the coatings are applied. Due to the drum rotation the coating machine can deposit single, bi- and multi-layers. The definition of the final coating recipe is still being developed [15]. To that end we have, together with DTU, recently stabilized the single layer iridium deposition [16] and are now working to develop a high-Z/low-Z bi-layer structure to further boost the effective area of the optics. Once this has been completed, we can proceed to testing multi-layer coatings to possible further improve the effective area of the ATHENA optics at $7 \mathrm{keV}$.

After un-loading the coated plates they are put back into cleaning containers and again loaded into the automated wetbench, where a lift-off process removes the resist covered area and dries the plates.

\section{Stacking}

The stacking process of the coated plates is carried out by fully automated robots [17], of which 4 are currently installed at cosine inside cleanrooms. The robots have in the last year undergone a significant harmonization and upgrade process. This has resulted in a stacking time of about 6.5 minutes per plate (see figure 6), already in line with the requirements of producing stacks for the ATHENA optics. We have recently also upgraded one robot to be able to stack up to 76 plates, to test a possible simplification of the mirror module assembly process by having later to integrate only two stacks instead of four (see figure 5).
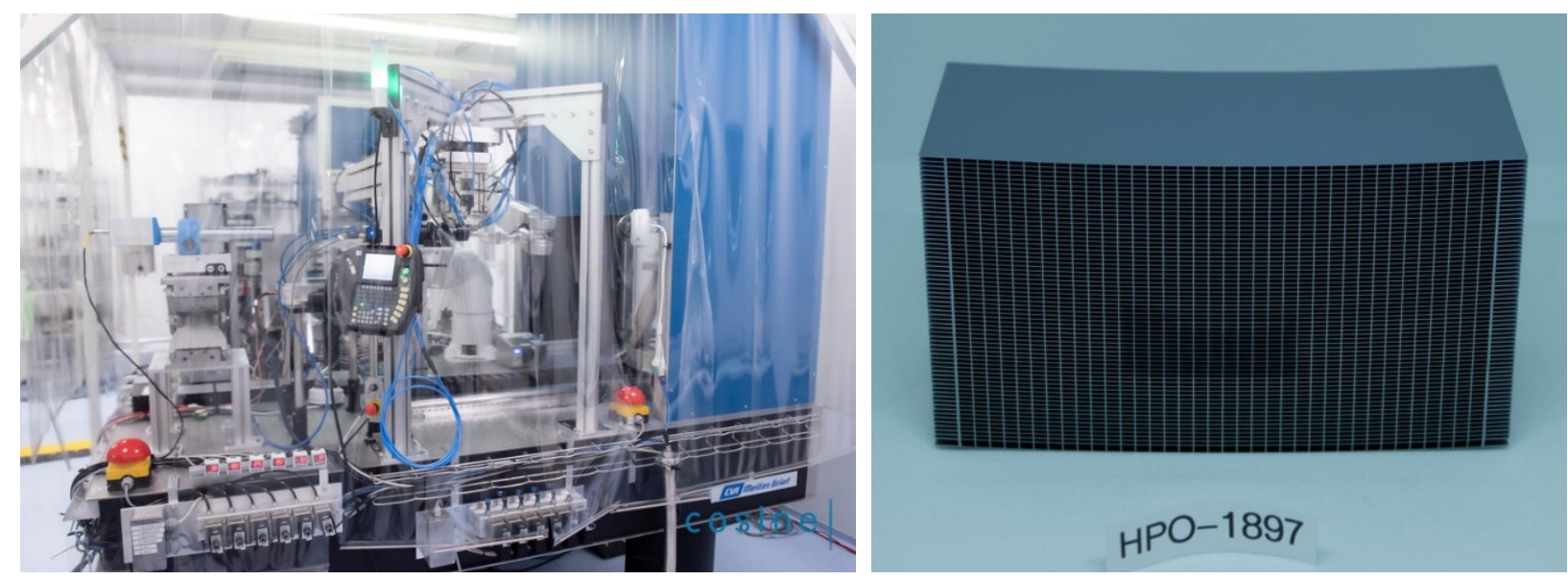

Figure 5: The left photograph shows one of the four stacking robots currently installed in one of the cleanrooms at cosine. The right picture shows a first prototype stack with a plate geometry that conforms to the latest ATHENA optical design (so-called row-08, with a $2.3 \mathrm{~mm}$ rib spacing and a $0.11 \mathrm{~mm}$ thin membrane), which consists of 67 plates stacked in an effort to develop and test the possibility of making 76 plates high single XOU mirror modules.
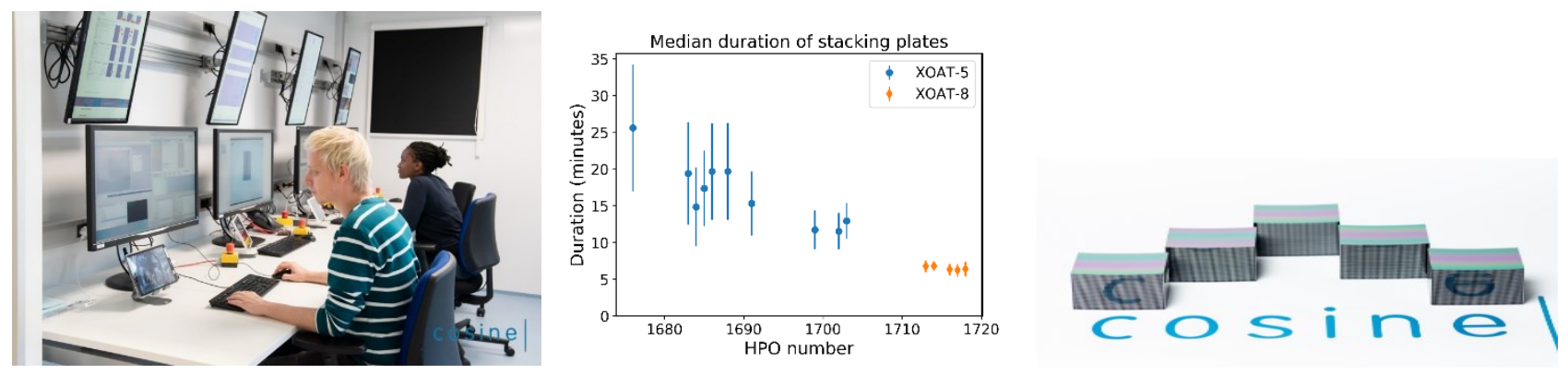

Figure 6: The stacking robots are remote controlled by laboratory technicians (left), who train producing stacks with repeatable quality in a reproducible timing. One of the earlier stacking robots (XOAT-5, middle) still included many manual steps, resulting in a variable stacking rate. XOAT-8, the newest stacking robot can stack plates with a cadence of $(6.5 \pm 0.8)$ minutes per plate. The resulting 5 stacks shown on the right, correspond to the 5 orange data points in the central figure. 
The harmonization of the robots not only allows exchanging components efficiently, but also reconfiguring a robot for a different plate geometry in a matter of days. Exchanging and aligning a mandrel (i.e. as required to make primary or secondary type stacks) can be done within an hour. The process control of the stacking process has been further tightened to electronically store all parameters in form of recipes that can be recalled by the operators when starting the stacking process. The stacking metrology that is generated during the stack production is automatically processed in real-time and used for X-ray performance prediction and comparison to other stacks. The performance prediction algorithms are continuously being further developed [18] based on the X-ray results we obtain from measurements at the XPBF 2.0 (Xray parallel beam facility) $[19,20]$, operated by PTB at the BESSY II synchrotron radiation facility in Berlin.

The post-stacking process steps have also been upgraded in the past year, as they start to take up a significant amount of time in the overall production schedule. The stacks undergo a thermal annealing process to increase the bond-strength, for which processes and procedures had to be developed. DTU also ascertained that the thermal treatment does not affect the iridium coating significantly [21]. The stacks then get a laser-marked ID to identify each stack uniquely, before being packaged and shipped for X-ray testing.

\section{X-ray testing and MM assembly}

X-ray testing of stacks and assembly of mirror modules is done in Berlin at XPBF 2.0 which has been upgraded in the last two years to operate at an energy of $1 \mathrm{keV}$ [22]. It also has received upgrades to improve the positioning accuracy and knowledge of the detector position with relation to the stack and mirror module coordinate system. We have also started to create additional cleanroom space next to the XPBF beamlines in an effort to increase the mirror module assembly rate, which requires storage of stacks, brackets and completed mirror modules at BESSY. The mirror module assembly process is being further developed [23, 24], as it is challenging to co-align 4 stacks to sub-arcsecond accuracy, especially with a desired production rate of 24 hours per mirror module. Significant effort has also been put into the automation of the Xray data analysis pipeline and the measurement procedures of stacks and pairs of stacks, called a X-ray optical unit (XOU). Those are now carried out by dedicated personnel and results of such measurements are usually available within 24 hours. To further increase our measurement capacity, ESA is in parallel developing an additional beamline at the ALBA synchrotron [25] near Barcelona, which will become available in 2022.

\section{PERFORMANCE IMPROVEMENT}

The status of the optics development is assessed and reported by measuring the X-ray performance. We routinely measure SPO test samples at the XPBF 2.0 beamline. Individual stacks of SPOs are co-aligned in double-reflection using direct Xray illumination with $0.1 \mathrm{~mm}$ wide X-ray beams that probe the entire surface of the optics with high-spatial resolution. Combining tens of thousands of such measurements allows to determine the overall point spread function (PSF) and to determine its HEW. We routinely also have optics measured at the PANTER facility operated in Munich by the Max Planck Institut für extraterrestrische Physik (MPE) to provide a cross-check and to be able to quantify the effective area and off-axis performance [26 - 28]. We also started to prepare for mirror modules measurements using the new BEATRIX beamline that ESA is preparing together with the Osservatorio astronomico di Brera [29, 30], which should become available later in 2021.

The stacking process development uses representative radii as proxy for the final ATHENA configuration, with the socalled middle radius $(\sim 750 \mathrm{~mm})$ being the most advanced. The inner $(\sim 250 \mathrm{~mm})$ and outer $(\sim 1500 \mathrm{~mm})$ radii are also being developed in parallel and lessons learned on each radius are transferred to other radii.

We are continuously improving the stacking recipe of the robots to address a number of error components identified in earlier stack generations [9]. We had observed systematic deterioration of the performance on the azimuthal outer edges of the stacks, reducing the usable and well performing width of an XOU and potentially forcing the scientists having to make a choice between large effective area or high angular resolution. We can now report that the underlying mechanism that let to these errors has been understood. The solution required development of new over-sized mirror plate types with sacrificial lateral extensions (SALEX) parts, that are present during the stacking process, but removed afterwards before the XOUs are being integrated into mirror modules. We also improved the stacking equipment to allow finer control of the deposition of the plate edges during the stacking. This has in combination let to a significant improvement of the HEW over $100 \%$ width of an XOU (see figure 7 right), where for the first time a 20 plates middle radius XOU (S/N 101) in double reflection performs better than 10" (9.4"). Figure 8 shows the encircled energy fraction for XOU-0101. From the recorded spot (left), the PSF can be deduced and the angular width can be determined as function of the encircled energy fraction (right). 
Also, smaller areas of that XOU-0101 show significant improvement compared to earlier generations. This was achieved by improving the adherence of the optics to the optical design, especially its meridional curvature. The combined target radius of curvature for an XOU is about $1.6 \mathrm{~km}$, resulting in a meridional sag of about $100 \mathrm{~nm}$, which over a mirror length of only $40 \mathrm{~mm}$ is complex to control and set. Too little curvature would limit the XOU HEW to about 5.6", the conical approximation to a Wolter-I system, too large curvature quickly deteriorates the performance further. Figure 7 on the left shows that with improved control over meridional curvature we have been able to pass on a $10 \%$ wide area over 10 plates of the same XOU the 4.3" barrier, again for the first time.
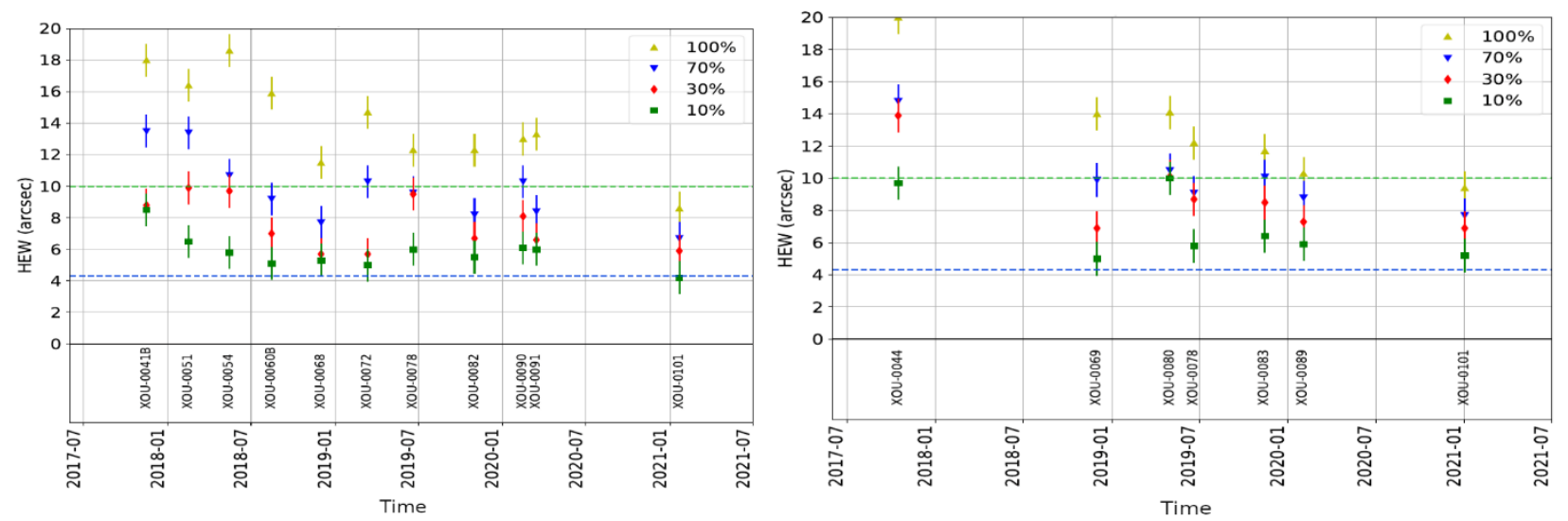

Figure 7: Evolution of the angular resolution (HEW) as a function of time, for 10 plates (left) and 20 plates XOUs (right).

Until 2020 all measurements are taken at $1.6 \mathrm{keV}$, as of 2021 we measure at $1.0 \mathrm{keV}$. The measurements report double reflection as measured at BESSY and the four sets of data points report different fractions of contiguous area width over 10 and 20 plates high scan areas. The gap in the middle of 2020 is related to COVID-19 related delays and supply issues.
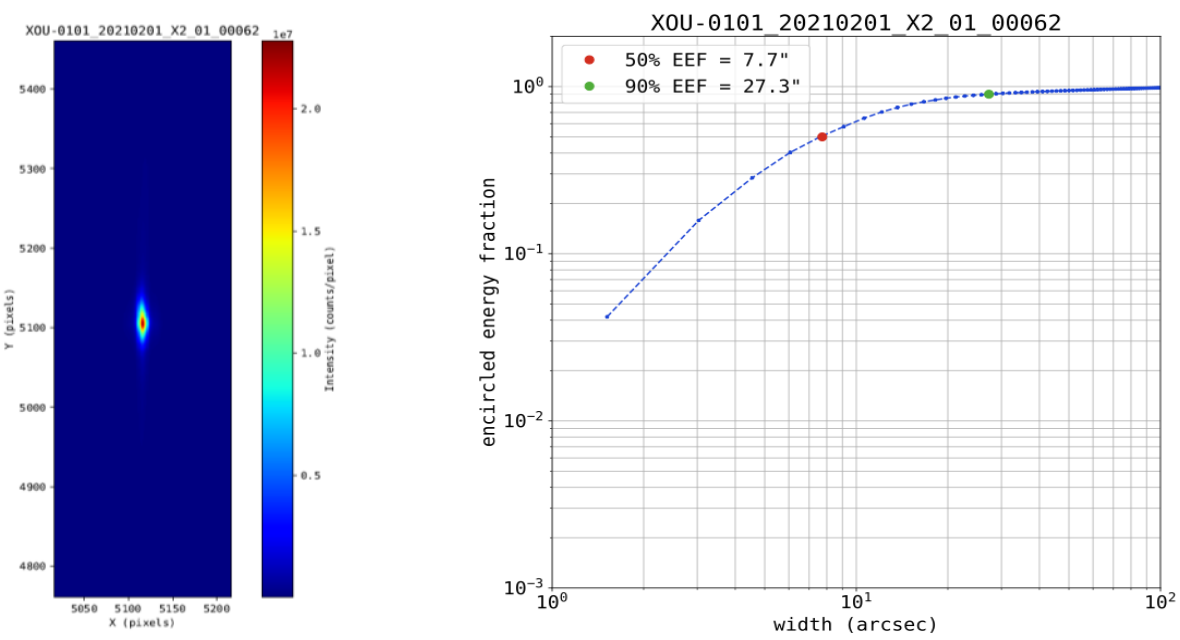

Figure 8: The PSF (left) and encircled energy fraction (right) for a middle radius XOU S/N 0101, for a 70\% wide contiguous area measured over 20 plates in double reflection at BESSY at $1 \mathrm{keV}$.

On outer radii we have, with an earlier generation stacking recipe which still lacks the SALEX improvements, also achieved a new bench mark, as shown in figure 9 . 

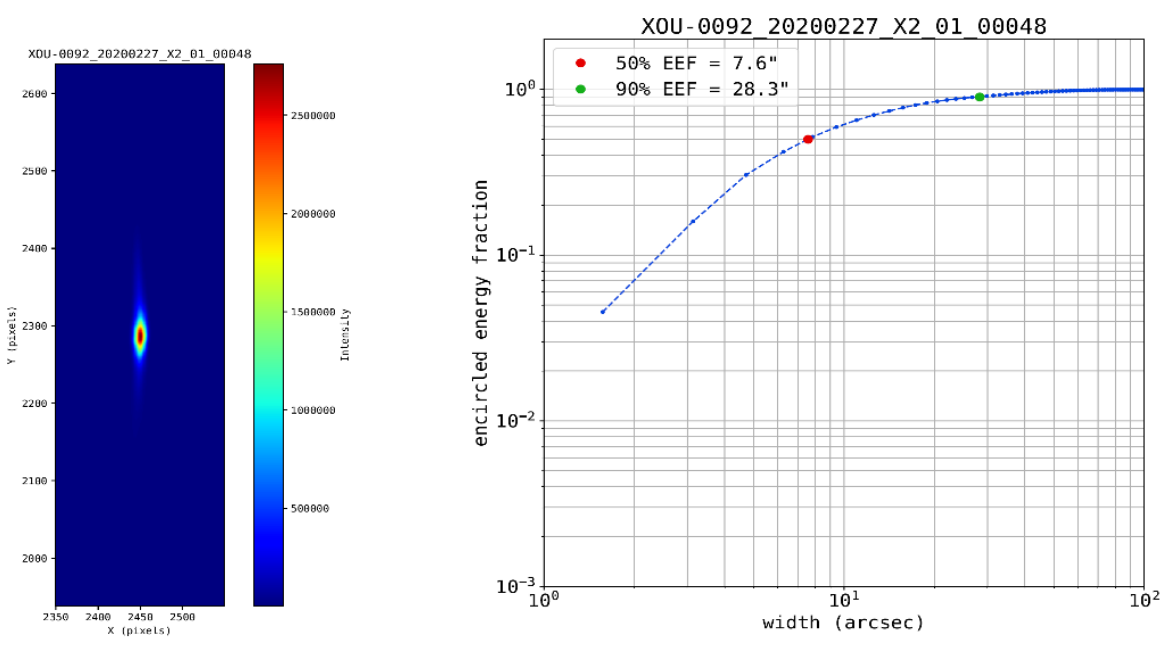

Figure 9: The PSF (left) and encircled energy fraction (right) for an outer radius XOU S/N 0092, for a 70\% wide contiguous area measured over 20 plates in double reflection at BESSY at $1.6 \mathrm{keV}$.

The lessons learned from the outer and middle radii stacking have also been applied to the inner radii stacking robot and in mid-2020 a performance of 16.9" was measured (see figure 10) over 34 mirror pairs, a significant single step improvement compared to the earlier measured 60 " performance.
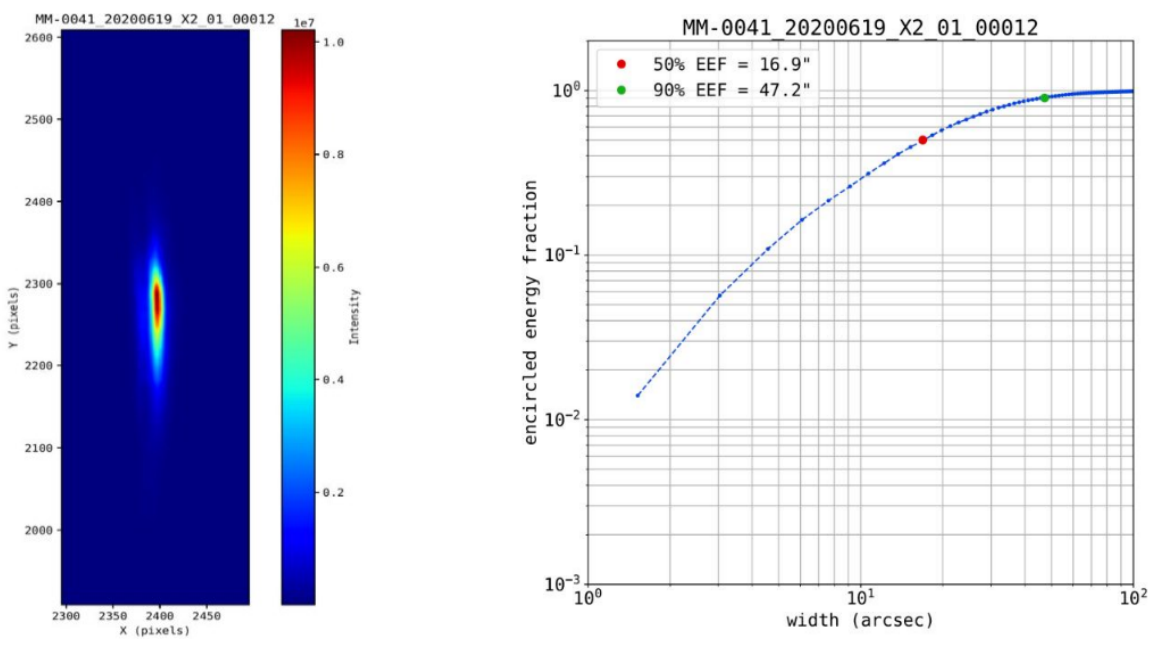

Figure 10: The PSF (left) and encircled energy fraction (right) for an inner radius XOU S/N 0094, for a 70\% wide contiguous area measured over 34 plates in double reflection at BESSY at $1.6 \mathrm{keV}$.

It shall be noted that XOU-0101 is the very first attempt at making stacks that use the improved generation 6 stacking recipes. In the course of 2021, we will continue to implement many improvements on both plate and stack level. The goal is to reach a 35 plates middle radius XOU performance significantly better than 10 " over $100 \%$ width and to push as large as possible an area below 7", as an important steps towards the ATHENA MAR.

The stack quality is being further improved through further development of the stacking recipe, which depends not only on the type of tooling used to pre-shape the mirror plate prior to bonding, but also on the timing and sequence of releasing the plate. Significant resources are being used to develop finite element models of the stacking process. The models are used to study the influence of plate parameters (e.g. oxide thickness and stress, membrane thickness, rib spacing etc) and 
of stacking robot parameters on the stack quality in terms of meridional curvature, edge effects and stack-up error. One example is shown in figure 11. Nevertheless, simulating an entire stack is still prohibitive in terms of computing time and we continue following the experimental route to improve the angular resolution step-by-step. The results, as shown in the HEW evolution (figure 7) show that optics quality keeps improving.
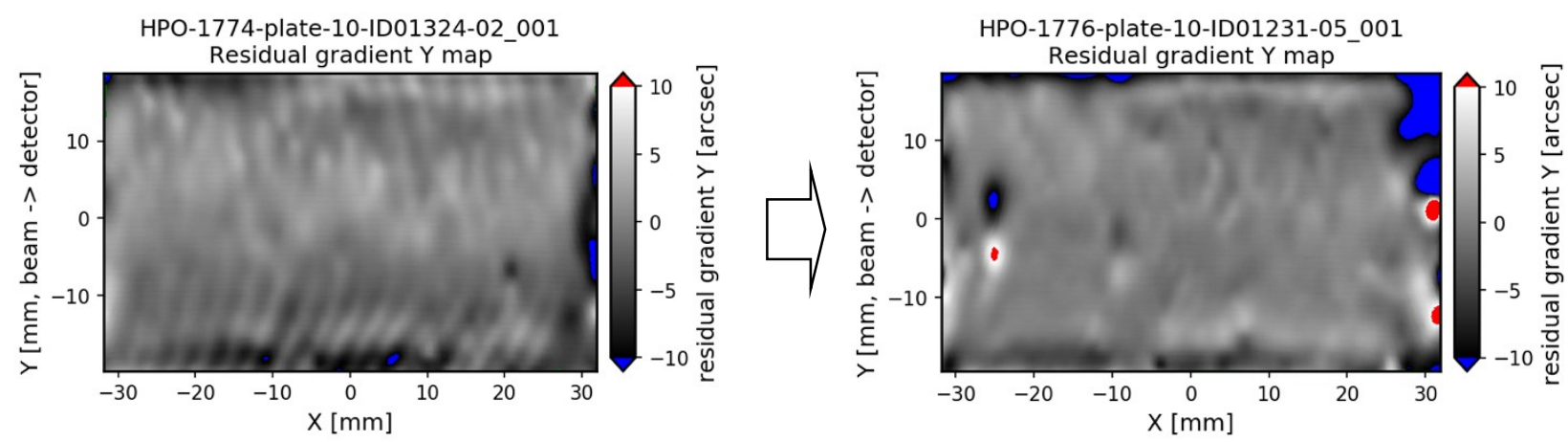

Figure 11: Example of the interaction between finite element modelling of the stacking process and improvements in practice. From the models a periodic structure was predicted to originate with a specific set of stacking parameters. The models predicted that those periodic structures could be reduced if the stacking robot would perform a smoother motion of the mandrel during the stacking process. Those changes were implemented and the stacking metrology data shows the before (left) and after (right) result of that particular process improvement.

\section{CONCLUSION}

The Silicon Pore Optics technology for ATHENA is being advanced on all fronts, as required to be able to pass the next mission gate, the Mission Adoption Review. We have shown that both the mass-production and the performance aspects of the optics are being developed by all partners with high speed. All process and production steps, from wafer up to mirror module level are being worked on, to ensure that the ATHENA optics can be built in-time and to quality.

\section{ACKNOWLEDGMENTS}

We would like to acknowledge all of the excellent and hard work of the many people and institutions that contribute to the development of Silicon Pore Optics and especially the contribution of the laboratory technicians of cosine. Without their efforts, dedication and talent we would not be able to build the lens required for the largest X-ray observatory to be flown so far. We also like to acknowledge the ESA, NWO (ATHENA, 184.034.002) and MPE funding that makes the development of SPO possible.

\section{REFERENCES}

[1] K. Nandra, "ATHENA: Exploring the Hot and Energetic Universe", Proc. American Astronomical Society HEAD meeting (\#14), (2014).

[2] Ayre, M.. et al., "ATHENA: phase A study status and optics/instrument accommodation", Proc. SPIE 11119, Optics for EUV, X-Ray, and Gamma-Ray Astronomy IX, 111190R (9 September 2019).

[3] Meidinger, N., et al, "The Wide Field Imager instrument for ATHENA", Proc. SPIE 9905, Space Telescopes and Instrumentation 2016: Ultraviolet to Gamma Ray, 99052A (18 July 2016)

[4] Barret, D., et al, "The ATHENA X-ray Integral Field Unit (X-IFU), Proc. SPIE 10699, Space Telescopes and Instrumentation 2018: Ultraviolet to Gamma Ray, 106991G (31 July 2018)

[5] Ferreira, I., et al., "ATHENA reference telescope design and recent mission level consolidation", these proceedings

[6] Willingale, R. et al, "Science requirements and optimization of the silicon pore optics design for the ATHENA mirror", Proc. of SPIE Vol. 9144, 91442E (2014) 
[7] Bavdaz, M., et al., "ATHENA X-ray optics developments and accommodation", these proceedings

[8] Vacanti, G., et al., "On the optical design of a large X-ray mirror based on silicon pore optics", these proceedings

[9] Collon, M. et al, "Status of the silicon pore optics technology", Proc. SPIE 11119, Optics for EUV, X-Ray, and Gamma-Ray Astronomy IX, 111190L (12 September 2019)

[10] Wille, E., et al, "Silicon pore optics manufacturing plan and schedule for ATHENA", Proc. of SPIE Vol. 10699, 106993L (2018)

[11] Valsecchi, G., et al., "Facility for alignment, assembly, and integration of the SPO mirror modules onto the ATHENA telescope", these proceedings

[12] Landgraf, B., et al, "Development and manufacturing of SPO X-ray mirrors", Proc. SPIE 11119, Optics for EUV, X-Ray, and Gamma-Ray Astronomy IX, 111190E (30 October 2019).

[13] Massahi, S., et al, "Installation and commissioning of the silicon pore optics coatings facility for the ATHENA mission", Proc. SPIE 11119, Optics for EUV, X-Ray, and Gamma-Ray Astronomy IX, 111190F (9 September 2019).

[14] Girou, D., et al, "Plasma etching for the compatibility of thin film metallic coatings and direct bonding of silicon pore optics", Journal of Applied Physics. 128, 9, 7 p., 095302

[15] Massahi, S., et al., "The effect of deposition process parameters on thin film coatings for the ATHENA X-ray optics", these proceedings

[16] Svendsen, S., et al., "Compatibility of iridium thin films with the silicon pore optics technology for ATHENA", these proceedings

[17] Keek, L., et al, "Stacking of mirrors for silicon pore optics", Proc. SPIE 11119, Optics for EUV, X-Ray, and Gamma-Ray Astronomy IX, 111190H (9 September 2019).

[18] Vacanti, G, et al., "X-ray testing of silicon pore optics", Proc. SPIE 11119, Optics for EUV, X-Ray, and GammaRay Astronomy IX, 111190I (9 September 2019).

[19] Krumrey, M., et al, "X-ray pencil beam facility for optics characterization", Proc. SPIE 7732, 773240 (2010).

[20] Krumrey, M., et al, "New X-ray parallel beam facility XPBF 2.0 for the characterization of silicon pore optics", Proc. SPIE 9905 (2016).

[21] Henriksen, P. L., et al., "Impact of annealing on performance of X-ray mirror coatings for ATHENA", these proceedings

[22] Handick, Evelyn, et al, "Upgrade of the X-ray parallel beam facility XPBF 2.0 for characterization of silicon pore optics", Proc. of SPIE Vol. 11444, 114444G (2020)

[23] Barrière, N. M., et al, “Assembly of confocal silicon pore optics mirror modules", Proc. SPIE 11119, Optics for EUV, X-Ray, and Gamma-Ray Astronomy IX, 111190J (10 September 2019).

[24] Barrière, N. M., et al., "Assembly of confocal silicon pore optics mirror modules", these proceedings

[25] Heinis, D., et al, "X-ray facility for the characterization of the ATHENA mirror modules at the ALBA synchrotron", Proc. of SPIE Vol. 11852, 1185222 (2021)

[26] Burwitz, V. et al., "Developing, testing, and calibrating the ATHENA optics at PANTER", Proc. SPIE 10399, 1039900 (2017).

[27] Bradshaw, M., et al, "Developments in testing X-ray optics at MPE's PANTER facility", Proc. SPIE 11119, Optics for EUV, X-Ray, and Gamma-Ray Astronomy IX, 1111916 (9 September 2019)

[28] Bradshaw, M., et al., "Effect of particulate contamination on a silicon pore optics", these proceedings

[29] Salmaso, B., et al., "Building the BEaTriX facility for the ATHENA mirror modules X-ray testing", these proceedings.

[30] Spiga, D., et al., "Performance simulations for the ground-based, expanded-beam X-ray source BEaTriX", these proceedings 